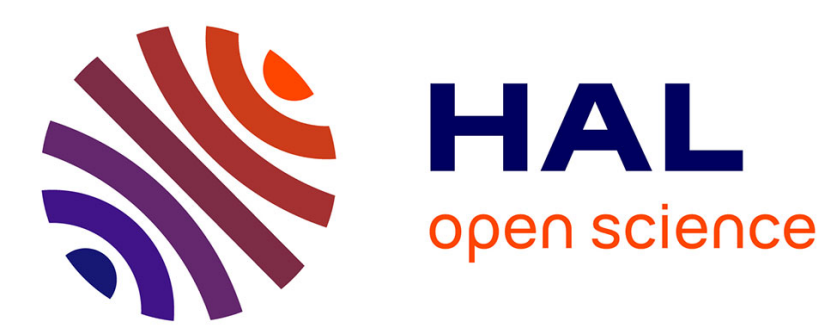

\title{
La nécessaire adaptation des modèles de coûts des directions des systèmes d'information
}

Isabelle Lacombe

\section{To cite this version:}

Isabelle Lacombe. La nécessaire adaptation des modèles de coûts des directions des systèmes d'information. Gérer et Comprendre. Annales des Mines, 2018, 3 (133), pp.25-39. hal-01885640

HAL Id: hal-01885640

https://hal-normandie-univ.archives-ouvertes.fr/hal-01885640

Submitted on 25 Jan 2019

HAL is a multi-disciplinary open access archive for the deposit and dissemination of scientific research documents, whether they are published or not. The documents may come from teaching and research institutions in France or abroad, or from public or private research centers.
L'archive ouverte pluridisciplinaire HAL, est destinée au dépôt et à la diffusion de documents scientifiques de niveau recherche, publiés ou non, émanant des établissements d'enseignement et de recherche français ou étrangers, des laboratoires publics ou privés. 


\title{
La nécessaire adaptation des modèles de coûts des directions des systèmes d'information
}

\author{
Par Isabelle LACOMBE \\ Professeur chercheur (assistant) en finance, chef du département « Droit, finance, \\ contrôle » et Directeur académique du M2 « Audit et finance d'entreprise » à l'EM \\ Normandie, Laboratoire Métis
}

Les directions des systèmes d'information (DSI) et la maîtrise de leurs coûts constituent un avantage concurrentiel pour une banque. Une étude de cas sur la fusion de deux DSI d'un groupe bancaire français confirme les apports de la littérature : le besoin d'une méthodologie spécifique de calcul de coûts dans les DSI. Une modélisation Activity-Based-Costing (ABC), enrichie de " services techniques ", appuie les benchmarks et choix technologiques, et lie directement les coûts aux services clients qui les utilisent. En outre, l'ajout d'un niveau d'analyse des données comptables les rend lisibles pour les opérationnels et les enrichit, ce qui permet une meilleure répartition. Enfin, une gouvernance renforcée par des contrats de services, avec un besoin de transparence accru pour lequel la piste d'audit entre les ressources et les services délivrés est nécessaire, s'avère plus cruciale dans un contexte de transformation et de mutualisation de moyens.

Les banques font face à un double défi : elles doivent considérablement développer la transparence sur leurs coûts, notamment à cause du développement des suivis de solvabilité, et elles doivent aussi faire évoluer leurs infrastructures et leurs outils pour aborder la transition numérique et faire face à une concurrence féroce. Tout ceci a conduit à l'augmentation des coûts informatiques dont le poids par rapport au chiffre d'affaires ou au produit net bancaire est, dans la banque, le plus important de tous les secteurs économiques. L'outil informatique et les infrastructures associées peuvent, en effet, être considérés comme l'outil de production de la banque.

Le suivi et la modélisation des coûts informatiques comportent certaines spécificités du fait de la complexité et de la rapidité d'évolution des technologies. Plusieurs praticiens ont proposé des modélisations plus ou moins abouties et généralement inspirées de la méthodologie ABC. Pour montrer les possibilités d'adaptations de la méthode $A B C$ pour le suivi et le pilotage des coûts informatiques, l'auteur s'appuie ici sur l'analyse d'une étude de la fusion de deux DSI « infrastructure » d'un groupe bancaire français de premier plan. II montre aussi qu'il est nécessaire de prévoir les processus et la gouvernance pour animer la modélisation des coûts et réussir une transformation de l'organisation.

Dans une première partie, l'article présente le contexte et les spécificités du pilotage et de la modélisation des DSI du domaine bancaire. Puis, dans une deuxième partie, dans une logique réflexive de recherche-action, et même de Design Science Research Methodology (DSRM) (MEVELLEC, 2017), l'analyse détaillée de la modélisation des coûts de la DSI du groupe bancaire est présentée (projet étudié entre 2009 et 2016). Enfin une troisième partie met en exergue les bonnes pratiques 
de la gestion de ce projet et de la conduite du changement, qui mènent à un bilan positif dont nous présenterons les leçons à tirer.

\section{Le contexte et les spécificités du pilotage et de la modélisation financière des DSI du domaine bancaire : une revue de la littérature}

\section{Le contexte économique et réglementaire du secteur bancaire entraîne un besoin accru de transparence et une gouvernance renforcée}

Le montant des coûts informatiques prend une part croissante du coût des organisations. Selon les différents secteurs, il peut représenter entre moins de $2 \%$ à plus de $7 \%$ du chiffre d'affaires ou du produit net bancaire $\left(\mathrm{PNB}^{1}\right)$. Ainsi il représente plus de $6 \%$ du PNB du secteur financier en 2012 (FORRESTER, 2012).

L'information et l'outil informatique constituent, en effet, les outils de production dans une banque.

Le développement du numérique et des services bancaires sur internet (on évoque un E-Business Model (SAHUT et LANTZ, 2011)) entraîne des coûts très importants d'infrastructures technologiques (ordinateurs, bureautique, serveurs de bases de données, maintenance informatique, équipement réseaux, etc.) qui se doivent d'être analysés.

L'utilisation de la monnaie unique en Europe et l'intégration de plus en plus forte des marchés financiers et du secteur bancaire ont entraîné de nombreux projets informatiques d'envergure internationale liés à l'automatisation des chaînes de traitement - le développement du Straight-Through-Processing (STP) dans le domaine de l'automatisation de la gestion des virements transfrontaliers et le développement du « Numéro de compte bancaire international » et de l' «Ordre international de paiement » ont conduit à des réflexions sur l'architecture du système d'information associé (DE LIMA, 2005). Tout ceci explique l'importance qu'accorde la banque aux DSI.

Le contexte économique et réglementaire actuel conduit également les banques à renforcer le développement de leur système d'information. Les banques se doivent d'être plus transparentes sur l'allocation des fonds : la BIS (Bank of International Settlement), au début des années 2000, a établi que les banques se devaient d'informer plus précisément leurs clients sur l'allocation des fonds et sur l'analyse des risques et de la solvabilité, ce qui permet de développer la qualité et de la fiabilité de l'information financière (SAIDANE et MECHRI, 2011). Le pilotage par les risques, prôné par les accords de Bâle et leur pilier 3, mène à publier des rapports périodiques, notamment sur internet, et à renforcer la gouvernance. "Certains banquiers vont jusqu'à proclamer ouvertement que la valeur de leur banque est celle des données dont elle dispose. Les données dans une banque sont omniprésentes et constituent la matière première pour de nombreuses activités : le trading des

\footnotetext{
1 Le PNB permet de mesurer l'activité d'une banque, il peut être assimilé à la notion de Chiffre d'Affaires dans d'autres secteurs.
} 
valeurs, la connaissance des clients, le reporting financier interne et externe, etc. » (LAVAYSSIERE, 2015).

La direction des systèmes d'information (DSI), par les services qu'elle fournit, peut apporter un avantage concurrentiel à la banque. La théorie du Resource Based Value est une approche « ressources et compétences » dans laquelle « la valeur provient de la détention par une entreprise de spécificités qui lui confèrent un caractère unique. L'avantage concurrentiel de la firme réside dans sa capacité à valoriser un ensemble de ressources internes au travers de compétences, entendues comme la faculté de coordonner ces ressources pour en développer une activité socialement responsable procurant un avantage concurrentiel ». Des auteurs vont même jusqu'à citer la réflexion menée sur les coûts de l'entreprise, comme par exemple la mise en place d'une comptabilité analytique ou d'une analyse de rentabilité des investissements, comme un avantage concurrentiel (EZZI et JARBOUI, 2016) et (DANNON, DUMOULIN et VERNIER, 2011). Cet avantage concurrentiel est décrit comme une « infostructure invisible » (CICHOWLAS, 2015) car il « s'agit de développer une infostructure qui est invisible pour les équipes informatiques, impliquant de gommer la complexité technologique, tout en restant cohérente et en ayant la possibilité de fournir des informations pertinentes, l'accent étant donc toujours mis sur les résultats opérationnels ». Différentes réalisations ont conduit les banques et leurs DSI à développer les nouvelles technologies et amorcer leur transformation numérique : l'utilisation d'ordinateurs génériques contrôlés par logiciel qui peuvent être configurés pour agir comme appareils de stockage de données un jour et comme serveurs le lendemain si nécessaire, ou encore l'utilisation des capteurs pour gérer à distance ses distributeurs de billets en surveillant les lecteurs de cartes, le stock d'espèces et l'alimentation électrique.

Les systèmes d'information contribuent à la performance d'une entreprise et doivent donc s'inscrire dans leur stratégie et leur gouvernance. La compréhension et l'analyse des systèmes d'information peuvent s'avérer complexes, à l'instar des systèmes d'information de gestion dont l'analyse « impose donc une approche ellemême transversale et pluridisciplinaire (en effet, les systèmes d'information revêtent des dimensions techniques, stratégiques, organisationnelles, comportementales, sociologiques, etc.) » (ELIDRISSI D. et A., 2010). II faut également intégrer le fait que le système d'information n'est pas un système figé, il change, se transforme en assimilant les mutations technologiques, en s'adaptant à la stratégie et aux choix structurels des organisations.

Les systèmes d'information financiers font partie intégrante de ce mécanisme de gouvernance car ils permettent l'intégration des approches partenariales avec la prise en compte de critères financiers mais aussi non financiers dans la mesure de la performance et permettraient « un enrichissement du cadre de la gouvernance, en sélectionnant les comportements vertueux au sein de l'organisation » (HASNAOUI et BIOT-PAQUEROT, 2010). Cela permet de développer la confiance entre les acteurs en intégrant les attentes des différentes parties prenantes.

La gouvernance dans le secteur bancaire comprend certaines spécificités comme un niveau de régulation et de surveillance plus important, une structure du capital spécifique, la prise en compte de la notion de risque, un besoin de garantie sur les capitaux, ce qui a pour résultat des comités de direction de taille plus importante et 
se réunissant à des périodicités plus rapprochées que dans le secteur industriel (AYADI et BOUJELBENE, 2013).

\section{Des recherches de modélisation adaptées aux spécificités des DSI}

Dans ce contexte de croissance des coûts informatiques dans les banques, avec le besoin d'une gouvernance accrue et d'un suivi des coûts plus transparent, les DSI ont éprouvé le besoin de disposer de modèles adaptés à leurs spécificités. Les DSI ont adopté différents référentiels de suivi de leurs activités, processus et services, comme les représentations ITIL, COBIT, CMMI ou Compass permettant notamment de servir de base de référence pour se comparer (on utilise le terme de « benchmark »). Les DSI se sont également tournées vers l'approche ABC pour valoriser les processus, activités et services fournis.

La méthode $A B C$ est née de travaux de chercheurs américains (JOHNSON et KAPLAN, 1987) et a d'abord été mise en place dans l'industrie sous l'égide du CAMI, Computer Aided Manufacturing-International (CAM-I, 1990). La méthode s'est ensuite développée dans le secteur des services et des activités de support, qui comprenait les DSI et les activités informatiques, avec des expériences dans le début des années 1990 (LACOMBE, 1997) (Commissariat général du Plan, Groupe présidé par Jacques Barraux, 1997).

Les activités informatiques ayant leurs propres spécificités, des groupes privés d'intérêt ou de recherche ont publié des travaux afin d'essayer d'adapter la méthode ABC aux DSI. On peut notamment citer les travaux du CIGREF (Club informatique des Grandes Entreprises françaises) qui a rédigé un rapport sur un modèle de référence permettant aux DSI de se comparer (CIGREF, octobre 2009). Un ouvrage présentant diverses études de cas de modèles économiques appliqués à des DSI sur des modèles de type CIGREF a montré l'intérêt d'utiliser l'ABC pour le pilotage des coûts informatiques (TREYER et BROGNIART, 2010).

Le modèle a connu différentes versions et évolutions, notamment en 2009 et 2014 (CIGREF, 2014), ce qui montre que les recherches et les praticiens ont eu besoin d'adapter les concepts de l'ABC aux spécificités des DSI. On notera par exemple que, dans sa version 2009, le modèle a cherché à s'appuyer sur les concepts de l'ABC, mais ses auteurs voulant démontrer qu'une adaptation était nécessaire, ils ont représenté le modèle à l'envers comme l'illustre la Figure 1 (les ressources en bas alors qu'elles constituent le point de départ en $A B C$ tel que représenté dans la littérature en sciences de gestion où elles sont, par convention, positionnées en haut du schéma avant les activités et avant les objets de coûts).

Figure 1 : Le modèle $A B C$ en trois niveaux tel que représenté par les travaux du CIGREF (version 2009). 


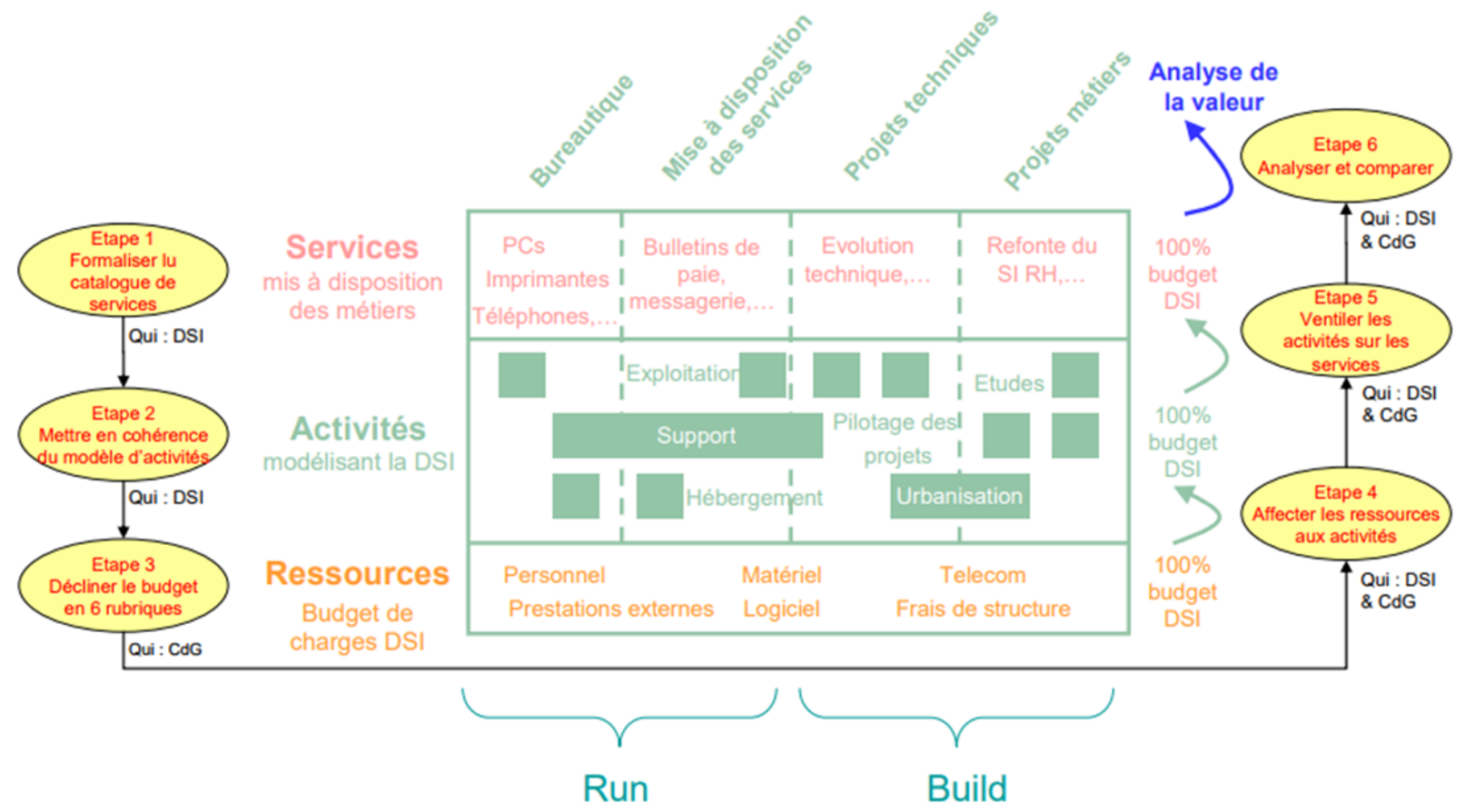

Source : CIGREF.

Le modèle des coûts informatiques du CIGREF comprend :

-4 processus,

- 39 activités en 2009 et 65 dans sa version 2014 (avec notamment la prise en compte des services liés au cloud),

- 6 rubriques budgétaires,

- des inducteurs d'activités (dans sa version 2014, le modèle du CIGREF applique la définition $A B C$ des inducteurs en prenant un inducteur unique par activité), - des indicateurs de pilotage et de benchmarking,

Enfin, le périmètre du modèle est la DSI hors MOA (maîtrise d'ouvrage) et AMOA (assistance à maîtrise d'ouvrage).

On notera que cette proposition a évolué dans sa version 2014 et n'est peut-être pas encore stabilisée. En effet les évolutions constantes des nouvelles technologies nécessitent de revoir régulièrement les référentiels (prise en compte du cloud et des modes Saas ${ }^{3}$, laas $\left.{ }^{4}\right)$. On peut évoquer à cet égard l'invention de la notion de "services techniques intermédiaires » permettant d'allouer les coûts en fonction de technologies et d'analyser et comparer des coûts génériques pour certains éléments (IT de la DSI, coût d'une plateforme de développement, etc.), ou encore le pilotage à

\footnotetext{
${ }^{2}$ Le cloud peut être perçu comme une externalisation des données, des applications et des services d'un client auprès d'un fournisseur. Ce fournisseur doit être distant et accessible via un réseau virtuel privé et/ou public (internet) (BOUAYNAYA et BIDAN, 2017).

${ }^{3}$ SaaS (Software as a Service) est un modèle d'exploitation commerciale des logiciels dans lequel ceux-ci sont installés sur des serveurs distants plutôt que sur la machine (PC en général) de l'utilisateur.

${ }^{4}$ laaS (Infrastructure as a Service) est l'une des trois principales catégories de services de cloud computing avec Platform as a Service (Paas) et Software as a Service (SaaS).
} 
l'aide d'une vision P\&L (compte de résultat) et d'une vision cash (encaissementdécaissement notamment au travers des activités sur les achats) au sein du même modèle. Nous noterons qu'en cela le modèle du CIGREF déroge à la méthodologie $A B C$ qui s'appuie sur une vision par le compte de résultat. Cette vision du CIGREF mêle deux modes de pilotage (gestion opérationnelle et investissement) et deux cycles différents (court terme et moyen terme) au sein du même modèle. Ces deux cycles et leurs impacts sur les comptes de la société doivent être expliqués aux décideurs ayant la possibilité d'engager des dépenses pour la DSI si l'on veut éviter d'éventuelles confusions.

D'autres recherches sur le calcul de coûts montrent qu'il peut être nécessaire de complexifier la modélisation de type ABC (WEGMANN, 2011) par les éléments suivants :

- L'utilisation de méthodes statistiques visant à fiabiliser les calculs.

- Une rationalisation de la phase d'allocation des ressources. La mise en œuvre de la comptabilité à base de consommation de ressources (RCA, Resource Consumption Accounting) est citée car elle revient à complexifier l'ABC puisque se combinent deux phases d'allocation, des ressources aux pools de ressources et des ressources aux activités, et que les éléments variables des éléments fixes sont systématiquement dissociés.

- Une analyse plus complexe des coûts informatiques. Une étude de cas indique qu'une forte externalisation des services complexifie le système d'allocation des ressources sur les activités et les services et qu'il convient d'intégrer fortement les partenaires au projet (logique de la gestion inter-organisationnelle des coûts). Dans ce domaine d'activité, des modèles de coûts plus complexes multiplient les métriques utilisées, les inducteurs « temps » n'étant pas suffisants pour répartir la diversité des ressources sur les prestations et les activités informatiques, ce qui permet de rassurer les clients, et donc de rendre moins discutables les propositions tarifaires.

Nous aurons compris le besoin de se pencher sur le détail et l'articulation de la modélisation des activités et services de la DSI afin de proposer un dispositif de pilotage adapté, ce dispositif comprenant la modélisation mais aussi la gouvernance associée. Notre question de recherche est : «En quoi est-il nécessaire d'adapter les principes de l'ABC et la gouvernance associée aux spécificités d'une DSI ? » Nous nous appuierons sur une étude de cas, la fusion de deux DSI infrastructure dans un grand groupe bancaire français. Cette étude de cas a été menée de juillet 2009 (avant le démarrage du projet) à mi-2016 (date du dernier entretien entre le chercheur et cette société). Le chercheur a été présent au moins à $50 \%$ de son temps au sein de cette société et a travaillé sur le projet notamment sur les aspects conceptuels, sur son cadrage et sur la mise en œuvre de son volet financier. La méthode de recherche s'apparente à de la recherche-action telle qu'elle a été décrite dans la présentation de l'impact des processus de recherche sur les organisations avec le cas du domaine " systèmes d'information » (MORLEY et BIA FIGUEIREDO, 2016). En gestion, la coproduction de connaissance s'inscrit souvent dans une méthodologie de recherche-action : "La réflexivité que le chercheur est capable de porter sur sa propre pratique est considérée de longue date comme un moyen privilégié d'améliorer la qualité et la pertinence des résultats de la recherche, en particulier lorsqu'il s'agit de recherches qualitatives. » Le chercheur peut, dans le cadre de la présentation de l'étude de cas, objet de cet article, garantir son 
indépendance et son objectivité dans la mesure où il avait préalablement déjà effectué de nombreux travaux de recherche sur le même thème depuis une quinzaine d'années, et avait déjà pu analyser une cinquantaine de projets $A B C$ dont une dizaine sur un périmètre d'activités informatiques. En outre, la période d'observation a été très longue et la taille de l'équipe impliquée très importante (une équipe-projet d'environ quarante personnes, avec pour moitié des acteurs extérieurs à l'entreprise). Ces différents acteurs ont, plus que le chercheur lui-même, pu influer sur le travail mené, laissant donc au chercheur une objectivité certaine. La méthode de recherche utilisée peut même plus largement s'apparenter à la Design Science Research Methodology (DSRM), dans la mesure où la recherche présentée ici vise " à trouver une solution idéale à un problème donné - par opposition aux méthodes traditionnelles qui visent, quant à elles, à comprendre le monde tel qu'il est » (MEVELLEC, 2017).

\section{Présentation de l'étude de cas sur la modélisation financière de la DSI infrastructure d'une banque}

\section{La présentation du contexte du cas}

En 2009, une des grandes banques privées de la place française, qui avait déjà mené de nombreux projets $A B C$ au sein du groupe sur des activités de type bancaire, a décidé d'appliquer cette méthode à sa DSI la plus importante. Cette banque avait en 2009 deux DSI infrastructure, une pour la banque d'affaires et l'autre pour la banque de détail. Elle disposait également de trois DSI métier qui travaillaient notamment sur le développement et la maintenance des applications de la banque d'affaires, de la banque de détail et d'une autre entité, filiale du groupe. Ces trois DSI consomment des coûts des DSI infrastructure. Malgré des spécificités techniques et des besoins propres à chaque domaine bancaire, la direction générale a décidé de fusionner les deux DSI infrastructure afin :

- de mutualiser certains moyens (par exemple des investissements en infrastructures) et prestations (des équipes projets et des méthodes de travail) afin d'assurer une continuité dans la qualité des prestations offertes mais également de pouvoir élargir la gamme des services pour ses clients internes, - d'atteindre une taille critique afin d'optimiser ses approvisionnements auprès de tiers de taille similaire, - de réduire ses coûts informatiques dans un contexte de contrôle des coûts du groupe.

La fusion des deux DSI infrastructure qui représentaient chacune, avant fusion, une part équivalente en termes de coûts (soit plusieurs centaines de millions d'euros chacune), formait une DSI de taille très importante :

- 2500 personnes et plusieurs centaines de millions d'euros de budget,

- 120000 postes de travail dans le monde, plus de 5000 applications, environ 50000 serveurs,

- de multiples services regroupés en sept familles :

- hébergement (datacenters, serveurs),

- connectivité (réseau et télécoms), 
- bureautique (postes de travail comprenant le matériel et les logiciels),

- services collaboratifs (messagerie, messagerie instantanée, visioconférence, etc.),

- support et expertise (notamment en matière d'architecture et de sécurité),

- projets liés à l'infrastructure,

- gestion des contenus ${ }^{5}$.

La création de la nouvelle entité, provenant de la fusion des deux DSI infrastructure, a connu de multiples contraintes :

- Des cultures très différentes entre la DSI de la banque de détail qui gère plutôt des activités de volume au travers des systèmes d'information des agences bancaires, et la DSI de la banque d'affaires qui prend davantage en compte les exceptions (par exemple les postes téléphoniques des traders qui sont différents de ceux des autres opérationnels).

- Des systèmes d'information hétérogènes, chaque DSI ayant développé ses propres outils. Ainsi, au total, il y avait trois systèmes différents de suivi des données sur les temps passés et chaque entité avait un système de messagerie avec des noms de domaines différents selon que le salarié était un employé de la banque de détail ou de la banque d'affaires.

- Des clients (banque de détail et banque d'affaires) qui possédaient leur propre modèle d'analyse de coût alimenté par celui de leur DSI d'origine, amenée à fusionner. Les modèles de coûts en question étaient des modèles de type $A B C$ : les directions financières et les directions des entités clientes étaient donc aguerries en matière d'analyse fine des coûts et avaient des exigences en termes de profondeur d'analyse et de formalisation des reportings fournis par la DSI.

Le projet de fusion des deux DSI infrastructure de ce groupe bancaire a été organisé en trois volets principaux :

- un volet « organisation et services » dont l'objet était de définir la nouvelle organisation, ses objectifs et la gouvernance associée,

- un volet financier dont l'objet était de définir le modèle financier (de type $A B C$ ) et les principes de gestion associés, et de les mettre en place dans un dispositif (outil et processus) à même de produire le détail des coûts par service et par client, de réaliser le budget sur ce même niveau de restitution, et de produire la facturation interne des coûts informatiques,

- un volet « données » dont l'objet était de définir les indicateurs et sources de données associées et de fusionner les outils en amont de ce dispositif financier (définitions détaillées d'extractions de l'outil comptable, des outils de suivi des temps passés, des outils de suivi des données techniques et opérationnelles comme les outils de suivi des immobilisations, de gestion des sous-traitants, de supervision des datacenters, etc.).

Le projet a été observé sur une période de sept ans :

\footnotetext{
${ }^{5}$ La gestion des contenus comprend notamment des logiciels destinés à la conception et à la mise à jour dynamique de sites Web ou d'applications multimédias.
} 
- De mi-2009 à fin 2010 : premières réflexions, définition des principes d'organisation, rédaction du catalogue de services informatiques, choix de l'outil $A B C$, mise en œuvre d'un pilote sur trois des sept familles de services.

- De fin 2010 à 2012 : déploiement de la nouvelle organisation et du modèle financier avec sa gouvernance associée.

- De 2013 à 2016 : utilisation du modèle et simplification de celui-ci (niveau de détail moins important et périodicité de calcul moins forte).

Ce projet présentait une démarche innovante car il comportait certaines particularités et difficultés.

Le périmètre du projet était très important, en termes de coûts comme en termes fonctionnels. Le projet coûtait plusieurs centaines de millions d'euros, trois fois plus que la plupart des projets sur les DSI. De plus, si un modèle $A B C$ sert à calculer les coûts, ici le projet devait également intégrer les aspects d'élaboration tarifaire (le pricing) et de facturation des prestations aux clients (le billing). En général, les projets intégrés se font dans des organisations matures et non dans des organisations en création comme pour le cas présenté.

Le pilotage du projet était bicéphale avec :

- Un chef de projet financier avec des objectifs de transparence et de traçabilité des coûts et de justification vis-à-vis des clients. II fallait donc disposer d'outils capables de présenter de manière très précise les déversements de coûts en partant de la comptabilité générale et en allant jusqu'aux offres de services et aux clients.

- Un chef de projet opérationnel IT avec des objectifs de compréhension des coûts des offres de services et des leviers possibles d'économie. Il fallait donc disposer d'outils capables de diffuser l'information auprès d'un public non financier sous un format compréhensible par lui.

Une dernière difficulté était liée au fait de mesurer une organisation en création dans le cadre d'une fusion.

\section{Une différence de modélisation nécessaire pour s'adapter aux besoins d'une DSI}

Les utilisateurs des services de la DSI (la banque de détail et la banque d'affaires) étaient très réticents quant à ce projet de fusion des deux DSI : ils craignaient de perdre en qualité de service (comment en effet comparer le poste de travail du trader à celui du personnel présent en agence bancaire ?) et ne souhaitaient pas pâtir de subventions croisées (la banque d'affaires ne souhaitait pas financer la banque de détail et vice versa). Il était donc nécessaire de mettre en place une modélisation de coûts très spécifique permettant une traçabilité des coûts et la présentation d'un niveau de détail très fin.

Dans un modèle $A B C$, tel qu'il a été présenté dans les travaux du CAM-I toujours valides à l'heure actuelle, il existe trois niveaux de modélisation :

- les ressources constituées généralement par la comptabilité, 
- les activités qui correspondent aux actions réalisées par les personnels de l'entreprise,

- les objets de coûts qui représentent la finalité du modèle et ce que l'on cherche à valoriser (en général les produits ou prestations dans le cas d'une DSI, les clients, etc.).

Cependant, dans le cas du projet mis en œuvre ici, le modèle a ajouté deux axes d'analyse supplémentaires:

- les modules de coûts : il s'agit d'un niveau intermédiaire entre les ressources et les activités,

- les BTO (ou Basic Technical Objects) qui représentent un niveau intermédiaire entre les activités et les objets de coûts.

La Figure 2 montre à quels niveaux le modèle a été enrichi : on y voit la différence entre un modèle $A B C$ classique et le modèle déployé dans la DSI qui a fait l'objet du projet.

Figure 2 : Différence entre la modélisation $A B C$ « classique » et le modèle $A B C$ de la DSI bancaire.
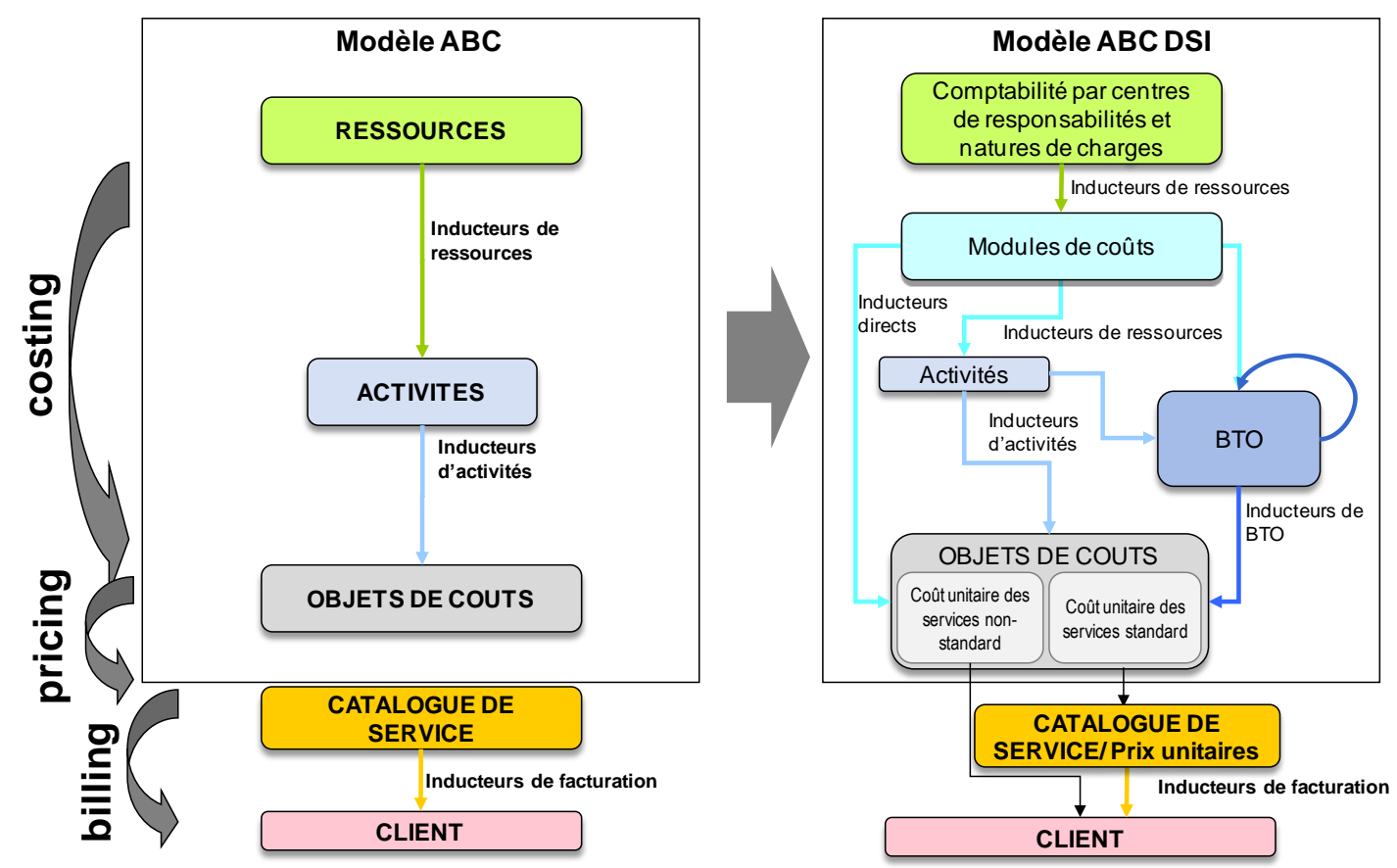

Source : Schéma réalisé dans le cadre du projet $A B C$ de la DSI faisant l'objet du cas étudié.

Des définitions ont été établies afin de préciser le contenu de chacun des niveaux du modèle et sont présentées dans la Figure 3.

Figure 3 : Définition du contenu des différents niveaux du modèle $A B C$ de la DSI bancaire. 


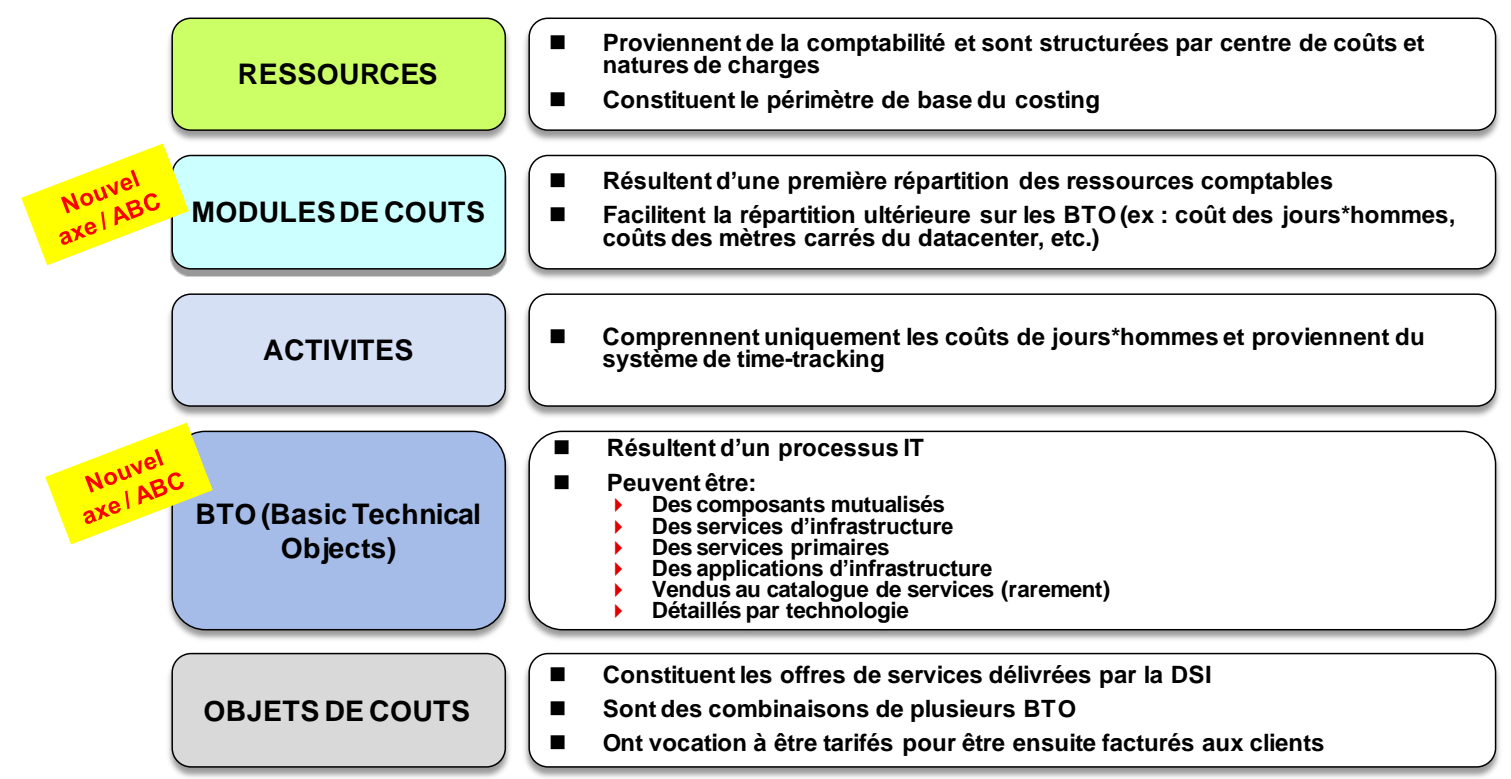

Source : Schéma réalisé dans le cadre du projet $A B C$ de la $D S I$ faisant l'objet du cas étudié.

Les deux niveaux supplémentaires, dont des exemples sont présentés dans la Figure 4, ont été créés pour répondre à des besoins d'analyse non couverts à l'époque.

Le niveau « modules de coûts » a été créé pour deux raisons.

Tout d'abord il a permis de présenter des notions comptables de manière générique aux informaticiens de la DSI. Ces informaticiens n'avaient pas toujours le temps, ni les prérogatives, pour s'intéresser à la façon dont la comptabilité était structurée par :

- Centres de coûts organisationnels : ceux-ci étaient parfois liés aux deux anciennes organisations et les modules de coûts ont permis de communiquer sur un niveau « anonyme » d'organisation. On pouvait ainsi s'intéresser au module de coûts datacenter sans s'intéresser précisément au fait que le datacenter provenait à l'origine de la DSI de la banque de détail ou de la DSI de la banque d'affaires. Par la suite, les datacenters ont fusionné et l'organisation initiale d'appartenance n'a plus eu d'importance.

- Natures de charges au travers des comptes comptables : ceux-ci étaient parfois trop peu détaillés ou trop peu lisibles pour permettre de retracer les coûts. Ces modules de coûts ont par exemple permis de regrouper les coûts de certains prestataires, de logiciels ou de matériels, en faisant le lien avec les outils d'achats ou de suivi des sous-traitants ou des fournisseurs.

De plus, ce niveau supplémentaire a permis d'établir une base de comparaison ou de regroupement entre les ressources de la banque de détail et la banque d'affaires, base découplée du contexte comptable de l'entité. II est à noter que les modules de coûts ne sont pas un niveau de consolidation, mais bel et bien un niveau sur lequel les « ressources » (la comptabilité) ont été réparties à l'aide d'inducteurs de ressources.

Le niveau « BTO » (Basic Technical Objects, ou éléments techniques basiques), a permis d'obtenir des consolidations au sein du modèle afin de comprendre la 
consommation de matériels ou de services primaires au sein de la DSI. Ces BTO composent les prestations. On peut noter que cet axe défini dans cette DSI en 2010 se rapproche de la notion de "service technique » présentée dans la version 2014 du modèle du CIGREF, ce qui confirme le besoin d'une vision technique propre aux besoins d'une DSI. Cet axe a ainsi notamment permis :

- De montrer des points de mutualisation de certains services élémentaires au sein de prestations. Cet aspect de mutualisation était crucial dans le cadre d'une fusion.

- D'avoir des éléments pour se comparer avec l'extérieur ou pour comprendre la formation du coût des prestations.

Figure 4 : Exemple de modélisation avec des modules de coûts (ressources) et des BTO.

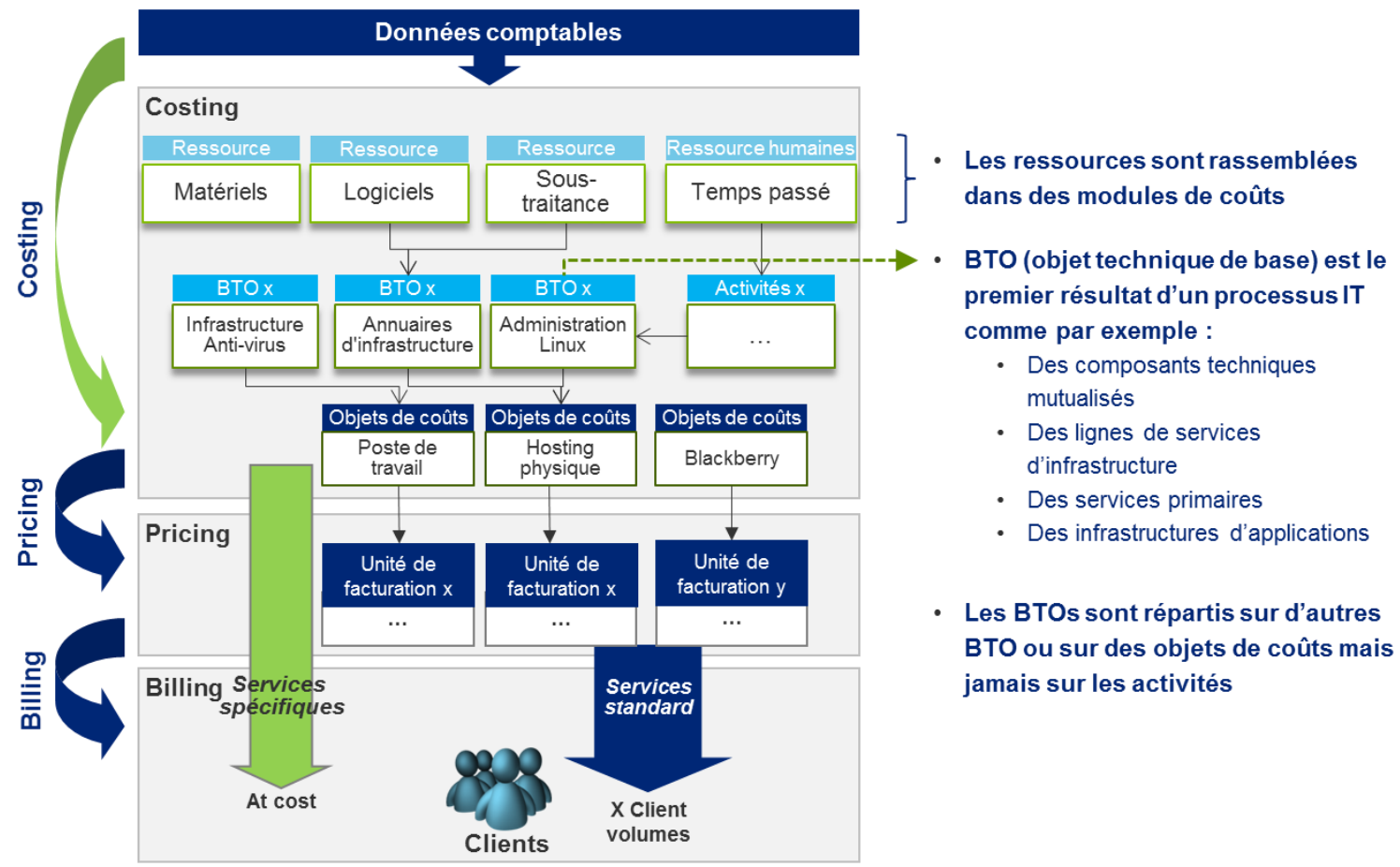

Source : Schéma réalisé dans le cadre du projet $A B C$ de la $D S I$ faisant l'objet du cas étudié.

Cette modélisation spécifique s'est d'abord basée sur les travaux de recherche et les études de cas publiées (CIGREF version 2009, etc.). Ceux-ci n'apportaient pas la réponse au cas étudié dans la mesure où ils étaient trop généraux dans un environnement où il était important :

- de prouver que la nouvelle organisation permettait d'engendrer des économies d'échelle au travers de la mutualisation de moyens entre deux organisations très différentes,

- d'apporter de la transparence sur les processus et les coûts et d'expliquer l'utilité de la nouvelle organisation en allant plus loin dans l'analyse des moyens techniques consommés.

Le modèle mis en place n'a pas présenté en tant que telles les notions de build (mise en place de nouveaux SI en tenant compte de la contrainte de les relier aux parties plus anciennes) et de run (coûts opérationnels de fonctionnement courant) présentes 
dans le modèle du CIGREF. Les objectifs du projet consistaient de prime abord à analyser les composants techniques et humains des services d'infrastructure de la DSI dans le but de les rationnaliser et d'en présenter les coûts au sein d'un catalogue de services. La modélisation a en quelque sorte pris pour hypothèse sousjacente que la majeure partie des coûts étaient du run présenté dans le catalogue de services. Les coûts de build étaient fondus dans le coût des services récurrents facturés (donc absorbés par les clients), intégrés dans les coûts de structure (là encore absorbés via les coûts des services), refacturés au siège pour les coûts de transformation majeure (donc neutralisés au sein du modèle), ou encore ils faisaient l'objet de prestations sur mesure facturées au prix coûtant (à l'euro l'euro) aux clients qui en faisaient la demande particulière.

\section{Les bonnes pratiques de gestion du projet et de conduite du changement ayant conduit à un bilan positif de la réorganisation}

Cette réorganisation de grande ampleur a fait l'objet de la création d'une direction de la transformation au sein de la nouvelle DSI. Cette direction a géré le programme de transformation en pilotant plusieurs projets (modification de l'organisation, création des nouveaux services, adaptation des outils informatiques de suivi de l'activité appelés « métrologie » et transformation des processus financiers).

\section{Des moyens conséquents pour une gestion de projet et une conduite du changement adaptées au fort besoin de transparence}

Ce projet avait un impact organisationnel très fort : les deux anciennes DSI infrastructure travaillaient chacune pour un seul métier et adaptaient leurs services aux demandes de ce seul métier. Ainsi, la DSI infrastructure de la banque de détail travaillait sur les postes de travail et réseaux utilisés en agence et au sein du réseau (distributeurs de billets, etc.) avec des volumes importants et une complexité relativement faible pour les postes en agence. La DSI infrastructure de la banque d'affaires travaillait sur les besoins plus spécifiques des traders et du métier de l'investissement, avec des équipements plus complexes et des coûts unitaires plus importants. La fusion des deux DSI avait pour but d'uniformiser les pratiques et les équipements et de faire en sorte que la nouvelle DSI devienne un centre de service financé par les deux métiers. Les moyens étaient donc mutualisés et refacturés aux clients internes. Ces clients craignaient que le nouveau modèle de coûts n'entraîne des subventionnements croisés : aucune des deux entités ne voulait financer l'activité de l'autre, et les deux souhaitaient un dispositif équitable. Le modèle de coûts développé avait pour but de formaliser un langage commun et une base partagée de contractualisation qui a pris la forme d'un contrat de service : la nouvelle DSI a formalisé une liste de services fournis au sein d'un catalogue avec pour chaque service :

- un descriptif du service fourni,

- un engagement sur des délais ou sur des résultats attendus,

- un prix proposé pour ce service,

- la possibilité de souscrire à des options en plus du service de base. Par exemple, si le service « poste de travail » était jugé inadapté par le client, la possibilité était 
offerte de souscrire à des options de type « double écran » ou « mémoire renforcée ». Ces options étaient facturées en supplément afin de responsabiliser les clients sur leur consommation.

Cette modélisation très précise des services et le coût associé à ceux-ci ont été les deux piliers permettant la réussite de la fusion des DSI. Ils ont permis en quelque sorte de contractualiser l'activité de la nouvelle entité vis-à-vis des clients. Afin de réussir cette opération, de nombreuses actions de conduite du changement ont été mises en place. Le projet a été structuré en étapes successives afin de mener la transition par phases. Ces différentes phases correspondent à celles du processus de transformation organisationnelle décrit dans la littérature (BESSON et ROWE, 2011) :

- Une phase de déracinement qui permet de « sortir de l'organisation ancienne, de s'en déprendre » : la nouvelle DSI a tout de suite été officialisée avec une organisation transitoire et le projet de fusion des processus des deux anciennes DSI a été mené sous cette nouvelle entité organisationnelle. Le nouveau DSI se devait donc de réussir cette fusion qui était demandée par le top management du groupe. - Une phase d'exploration / construction de la nouvelle organisation :

- Une étude au travers d'une centaine d'entretiens avec des opérationnels a permis de cartographier les processus et de concevoir le modèle de coûts (référentiels et clés de répartition ou inducteurs à utiliser). Ces entretiens ont réuni les personnes de terrain, qui se sont accordées sur un référentiel commun.

- La mise en œuvre d'un pilote de mars à juin 2010 sur trois des sept familles de services. Ce pilote a consisté à modéliser et documenter les services, collecter les données sur une période de référence, procéder au calcul des services et présenter les résultats pour analyse. Ce pilote a permis de déterminer le niveau de détail adapté (ni trop lourd, ni peu détaillé au point de nuire à l'analyse) et d'identifier les difficultés éventuelles (données non disponibles, désaccords entre les acteurs sur certaines règles). Ce travail a débouché sur un séminaire d'une journée réunissant une centaine de personnes, avec à la fois des décideurs et des opérationnels ou des financiers de la DSI et de ses clients, séminaire qui a permis de partager et de discuter les conclusions du pilote et a officialisé la fin de cette étape, permettant d'entamer les validations détaillées et la généralisation du dispositif.

- Une phase de stabilisation et d'institutionnalisation de l'organisation nouvelle avec une validation détaillée particulièrement importante : le modèle a été testé auprès des opérationnels. Les tableaux de résultats très détaillés présentant l'intégralité des détails de déversements leur ont été communiqués pour qu'ils vérifient la pertinence de la modélisation.

- Une phase d'optimisation et de routinisation avec :

- La généralisation du modèle. Une fois les corrections effectuées, l'ensemble du périmètre est déployé à partir de septembre 2010 sur les quatre autres familles d'offres de service.

- La simplification du modèle. Nous verrons plus loin que le dispositif projet et le modèle ont été simplifiés par la suite.

Un autre élément de conduite du changement et de gestion de projet ayant permis le succès de cette opération a été le fait de mobiliser une équipe bicéphale avec des opérationnels de la DSI et des financiers. Durant la phase de projet qui a duré environ trois ans (de fin 2009 à fin 2012), le projet a mobilisé environ quarante 
personnes (équipe projet et contributeurs) dont la moitié étaient externes. Ces quarante personnes étaient:

- des opérationnels de la DSI (des spécialistes des sept familles de services modélisés) qui ont participé à la définition du catalogue de services très détaillé dénombrant une soixantaine de services différents, à la spécification des inducteurs nécessaires à leur mesure et à la convergence des services, - des financiers de la DSI : le contrôle de gestion de la DSI a défini les nouveaux principes financiers sur le calcul de coûts, le mode d'élaboration du budget de la DSI et la tarification des services de la DSI (les services pouvaient être facturés avec une marge).

La conduite du changement a mobilisé de nombreuses personnes au travers d'une vingtaine d'ateliers de présentation des résultats détaillés du premier modèle. Divers états de restitution présentant le coût par service avec sa décomposition par ressource, activité et BTO, avaient été préalablement fournis. Ils permettaient de présenter la piste d'audit et de démontrer aux clients qu'ils étaient facturés d'un montant équitable (rappelons que leur crainte était de subventionner les autres clients de la DSI). Les clients du modèle ont demandé le développement d'un outil graphique décrit dans l'Encadré 1, afin de disposer d'arbres de décomposition des coûts (depuis les ressources jusqu'aux services). Cet outil a donné un accès très clair et très détaillé à la décomposition des coûts, ce qui a crédibilisé le modèle auprès des clients de la DSI. 


\section{Encadré 1 : Une représentation graphique des coûts :}

Le modèle de coûts a été calculé avec un outil standard de calcul de coûts du marché. Cet outil a cependant présenté certaines limites dans le cas du modèle de la DSI qui nous intéresse :

- II permettait de déverser correctement les coûts du haut vers le bas du modèle (des ressources vers les activités),

- II traitait correctement les boucles récursives de calcul en utilisant la méthode par itération. Ce type de boucles apparaît quand deux activités se déversent l'une sur l'autre.

En revanche, l'outil ne restituait pas la traçabilité des coûts de bas en haut (traçabilité qui permet de donner la part des ressources comptables consommées par chacun des objets de coûts (les prestations). En effet, l'outil globalisait le résultat des calculs des boucles rétroactives : il ne gardait pas en lecture directe la trace de chaque itération de calcul, rompant ainsi le lien précis entre les ressources de départ et les objets de coûts à l'arrivée. II était possible de remonter la chaîne de calcul niveau par niveau (des objets de coûts vers les BTO, des BTO vers les activités, des activités vers les modules de coûts, des modules de coûts vers les ressources), mais il était impossible de remonter d'un seul coup tous les niveaux (par exemple des objets de coûts vers les ressources).

Cette limite a créé de gros problèmes d'acceptation du modèle : à un moment donné, les opérationnels ne comprenaient pas que l'on ne puisse pas justifier les coûts.

L'approche étant nouvelle, les opérationnels étaient sceptiques sur les résultats produits par la nouvelle modélisation. II a été nécessaire de leur présenter de manière détaillée les différents déversements de coûts. Au départ, des tableaux de chiffres présentant les déversements entre deux niveaux ont été élaborés. Ces tableaux ne donnaient pas satisfaction car ils ne permettaient pas d'avoir la traçabilité complète du cheminement des déversements de coûts depuis les objets de coûts (les prestations de services) en remontant par les BTO, les activités, les modules de coûts puis les ressources (la comptabilité). L'équipe a donc lancé un groupe de recherche pour développer un outil graphique de présentation des résultats. Les outils standard de costing du marché n'offrent pas en effet cette possibilité. Cet outil permettait de présenter sous la forme d'un arbre de coûts les liens entre la ressource, les modules de coûts, les basic technical objects, les activités et les services du catalogue.

Après le développement de cet outil spécifique à l'aide de Visio pour la partie graphique, les opérationnels et contrôleurs de gestion ont pu interroger pleinement le modèle et obtenir la transparence souhaitée.

Chaque nœud de la représentation est constitué par les champs suivants :

- nom de l'élément (BTO, module de coût ou objet de coût),

- nom de l'inducteur permettant le déversement de cet élément sur ses éléments supérieurs,

- montant impliqué dans l'élément supérieur,

- montant global de l'élément, 
La comitologie associée à ce projet a été très stricte. Au début du projet et pendant un an, trois comités se réunissaient :

- Un comité par volet (organisation et services, finance, données) se réunissait toutes les semaines pour valider les options à prendre sur les différents thèmes.

- Un comité projet se réunissait toutes les semaines pour travailler sur les éléments débattus dans les comités par volet : choix de modélisation, résolution des problèmes (retards, difficulté d'obtention de certaines informations) et affectation des moyens au projet (prioriser les sujets et les affectations des équipes).

- Un comité de pilotage se réunissait pour valider les grands jalons : catalogue de services, principes financiers, choix de l'outil $A B C$, choix de modélisation, résultat des calculs.

Cette validation graduelle et de grande ampleur aux différents niveaux de management a été un facteur clé de l'acceptation du nouveau dispositif.

\section{Un bilan positif de ce projet avec un impact organisationnel important}

Ce projet a été mené à son terme et les différentes modifications de processus et d'organisation ont été mises en place.

\section{Une DSI organisée comme un centre interne de services s'appuyant sur un catalogue}

La mutualisation des deux DSI a pris la forme d'une DSI centrée autour de son catalogue de services. Le catalogue a décrit des services communs que les deux entités clientes pouvaient acheter. Ce catalogue organisé autour de sept familles de services a été animé par sept responsables d'offres. Cette nouvelle fonction de responsable d'offre a été créée afin d'assurer l'interface entre les clients internes (banque d'affaires et banque de détail) au travers de la collecte des besoins (évolution et volumétrie des services consommés par les clients), les opérationnels de la DSI (leur communiquer les besoins pour qu'ils puissent travailler sur l'évolution et la délivrance des services) et les financiers (les responsables d'offres participaient à la définition des coûts et du prix des services). La nomination de ces responsables d'offres a permis d'adopter un mode de management plus agile que l'on peut qualifier de « mode décentralisé et réactif » (CASEAU, 2011). En effet, ces responsables d'offre appartenaient, au sein de la DSI, à des équipes décentralisées et tournées vers les clients. Ils ont joué un rôle fédérateur majeur dans la réorganisation au travers de l'animation des offres présentées par le catalogue de services, qui permettait une contractualisation facilitée et une réduction des coûts de transaction (WILLIAMSON, 2002).

Ces responsables d'offre ont également analysé les coûts de sous-traitance : la mutualisation a permis de massifier les achats. Le but n'était pas de sous-traiter davantage en externe, mais de mieux piloter les fournisseurs. Les modules de coûts décrits dans le modèle $A B C$ ont permis aux opérationnels de voir en lecture directe la contribution des différents fournisseurs aux services fournis par la DSI, et ainsi 
d'optimiser leur utilisation. Certains services ont été délocalisés en Inde, mais auprès d'une équipe interne de la Banque. Le but était de s'appuyer sur des équipes aux coûts salariaux plus faibles.

Les DSI ont fusionné et les services ont donc eux aussi convergé : il n'y avait plus plusieurs postes de travail différents, mais un socle commun avec la possibilité d'y adjoindre des options faisant l'objet d'une facturation supplémentaire. II était donc toujours possible de disposer d'un double écran ou d'un poste plus puissant, mais c'était dorénavant plus cher pour l'utilisateur. Les datacenters ont été rationalisés, les postes de travail de plus en plus virtualisés ${ }^{6}$, les investissements lourds en infrastructure ont été mutualisés dans cette nouvelle organisation au sein d'un cloud privé. Ainsi, les DSI clientes (DSI métiers) ne consommaient plus un serveur dédié pour leur application mais commandaient des gigaoctets de stockage avec un certain niveau de disponibilité (le niveau de service gold leur assurait une maintenance $24 \mathrm{~h} / 24$ alors que le service bronze coûtait moins cher mais proposait des niveaux d'intervention plus longs). Ces nouvelles pratiques permettaient d'adapter les usages et de ne pas systématiquement exiger le niveau de qualité ou de service maximal.

Les membres des équipes travaillant sur cette nouvelle DSI provenaient des deux anciennes DSI. Elles ont été fusionnées pour travailler ensemble sur les nouveaux services. Elles ont notamment été jugées sur l'atteinte des indicateurs de qualité et de service présentés dans le catalogue de services (temps d'intervention, respect des délais, respect des engagements de service) et sur le respect des coûts qui forment la base du prix. Tout ceci a été permis par la mise en commun des systèmes de suivi. Ainsi, auparavant, il existait trois systèmes de mesure du temps passé au sein des deux DSI (encore appelés time tracking) : après la fusion, il n'existait plus qu'un seul outil.

\section{Un rôle structurant des processus financiers dans la réussite de la nouvelle organisation}

Trois processus financiers ont structuré la refonte de l'organisation : le costing, le pricing et le billing. Ils ont permis d'adopter un langage commun et d'apporter une transparence et un support à la contractualisation.

- Le calcul de coûts (costing) : le modèle $A B C$ a été alimenté et paramétré à l'aide d'un outil $A B C$ du marché par une équipe du contrôle de gestion. Le modèle était calculé tous les mois et permettait de répartir les ressources de la DSI sur les BTO et les activités, et de calculer le coût total, puis le coût unitaire de chacun des services. Ce costing a été un facteur déterminant de la convergence des services. Ainsi, comme le présente la Figure 5 , en début de projet, des états de synthèse ont permis d'analyser les différences de coûts pour un même service (ici le poste de travail) délivré par les deux DSI d'origine. Après analyse détaillée, les meilleures des deux pratiques ont été conservées afin de proposer des modes d'organisation et des processus aboutissant à un coût optimisé du poste de travail.

\footnotetext{
${ }^{6}$ La virtualisation consiste à disposer de postes de travail plus légers (au contraire des clients lourds) et d'installer les applications sur des serveurs distants et centralisés permettant une maintenance et une mise à jour centralisée et facilitée.
} 
Figure 5 : Exemple d'état de présentation de synthèse des résultats du modèle (décomposition des coûts des deux types de postes de travail par BTO et activité avant la convergence vers un poste de travail unique au sein du groupe).

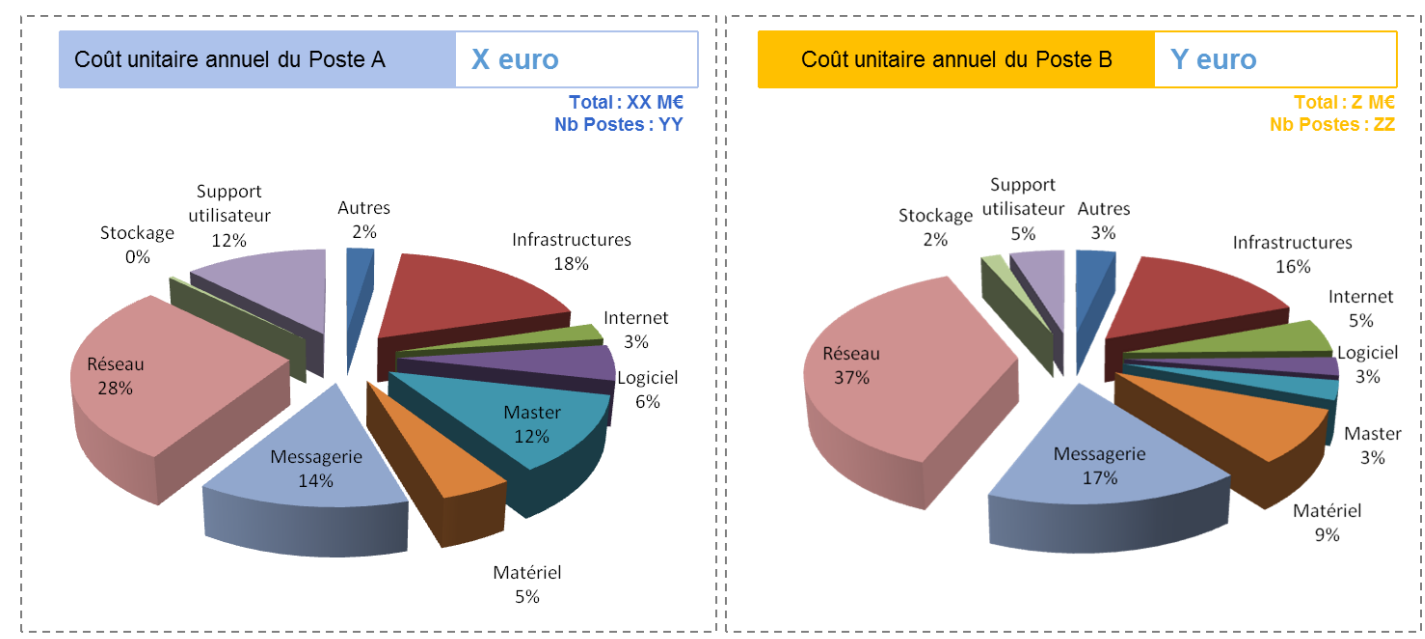

Source : État réalisé dans le cadre du projet $A B C$ de la DSI faisant l'objet du cas étudié.

- Le budget (pour le pricing) : chaque année, le modèle fait l'objet d'un calcul en mode budget avec une alimentation des volumes prévisionnels d'offres communiqués par les clients aux responsables d'offre. Cette simulation budgétaire permettait d'adapter la tarification des services dans la mesure où il fallait respecter l'équilibre « coûts de la DSI = facturations de la DSI » car la DSI, département interne au groupe, n'était pas autorisée à dégager de marge. Elle définit ainsi un prix pour chacun des services en fonction de la règle suivante : les services ne sont pas facturés à leur coût de revient, mais une politique de tarification incitative et dissuasive a été mise en place. Les services sont regroupés au sein de familles et chaque famille doit présenter un bénéfice nul. II est cependant possible, au sein d'une même famille, de proposer des services à un prix dissuasif supérieur à leur coût (cas des services matures dont les coûts sont amortis et que la DSI souhaite décommissionner ${ }^{7}$ ) ou à un prix incitatif inférieur à leur coût (cas de nouveaux services dont les coûts de développement ne sont pas encore amortis et pour lesquels les premiers utilisateurs peuvent se montrer réticents, craignant notamment de faire face à des réglages techniques ou à des adaptations dans la mesure où ces services n'ont pas encore été éprouvés par d'autres utilisateurs). Ce pricing a permis de dégager des ressources pour développer de nouveaux services et accélérer la transition numérique.

- La facturation (billing) : une équipe dédiée à la facturation se charge chaque mois d'établir la facturation interne des services pour les clients français et internationaux au sein du groupe. La facture présente le détail par service des volumes consommés et des prix unitaires. Elle provoque un effet vertueux car les clients peuvent établir le lien entre leur comportement de consommation des prestations informatiques et la

\footnotetext{
7 Le décommissionnement consiste à arrêter ou supprimer un service (matériel ou logiciel). Ceci engendre parfois des coûts quand il faut prévoir des actions de recyclage ou de démontage des matériels démantelés ou une migration vers le nouveau service (dans le cas de logiciels ou d'applications par exemple).
} 
facturation qui pèse sur leurs départements. Le but est, à terme, de responsabiliser les clients et de réguler la consommation, donc de réduire les coûts.

\section{Un allégement progressif du dispositif}

Si les besoins d'analyse et de justification étaient très fins en début de projet, une fois le déploiement de la nouvelle organisation et des nouveaux services effectué, il n'était plus utile de maintenir un dispositif aussi important.

Une fois le modèle déployé, l'équipe en charge de son animation et de sa maintenance s'est réduite à une douzaine de personnes (4 au sein du service financier pour alimenter l'outil et faire évoluer les règles, 7 responsables d'offre, 1 responsable de la facturation et des relais au sein des équipes opérationnelles), au lieu des 40 personnes mobilisées sur le projet.

Si le modèle était calculé tous les mois à un niveau très fin dans les trois premières années du projet, une fois le modèle compris et accepté par les clients internes et par les opérationnels, des actions de simplification ont été entreprises. Ainsi, à partir de 2013, le modèle a été allégé (regroupement de certaines activités par exemple) et la périodicité de calcul est passée au trimestre. Cela a permis d'alléger la charge de travail des équipes et de réduire les coûts de maintenance de ce modèle.

Le dernier entretien mené en 2016 avec cette société indique que le modèle est toujours utilisé et qu'il est maintenant maîtrisé. Les coûts informatiques sont toujours facturés sur la base des informations produites par celui-ci. Cette longévité de sept ans confirme le succès de ce projet et la pertinence de la modélisation adoptée. Les indicateurs de gestion, s'ils sont toujours basés sur les coûts, la qualité et les délais, sont désormais articulés autour des services du catalogue, basés sur des définitions communes et mesurés par des outils communs à la suite de la mutualisation et de la simplification des outils de mesure. Concernant le bilan de la réorganisation, il est plutôt positif. Si certains acteurs ont choisi assez tôt de quitter l'entreprise face à l'ampleur des modifications de leur travail habituel, cela a plutôt été le cas pour des managers qui perdaient du pouvoir dans la nouvelle organisation. Les opérationnels de terrain ont globalement accepté cette transformation qui allait de pair avec une professionnalisation et une modernisation des processus. Quant aux aspects économiques, la réorganisation a entraîné des surcoûts non prévus au départ mais a fini par engendrer globalement des économies substantielles.

\section{Conclusion}

Les recherches bibliographiques et l'étude de cas présentée convergent toutes sur le fait qu'il est nécessaire de développer une méthodologie spécifique de la modélisation des coûts dans les DSI, surtout dans le domaine bancaire. La réussite de ce type de modélisation implique de mobiliser des équipes pluridisciplinaires alliant des financiers, des spécialistes des systèmes d'information et des garants de la qualité des données. Les clients des DSI sont particulièrement exigeants : ils sont dans le même groupe, mais ont accès à des benchmarks ou des comparaisons de coûts informatiques (il suffit de se rendre dans un magasin informatique pour consulter le prix d'un PC). Mais la réalité est plus complexe car un PC, ce n'est pas 
seulement une machine, c'est aussi une infrastructure associée et des couches logicielles qu'il faut en outre maintenir. Pour répondre à des objectifs de

transparence, les DSI du monde bancaire ont dû développer les axes d'analyse et de modélisation de leurs coûts. Les modèles étudiés dans les recherches

bibliographiques et celui du cas présenté démontrent qu'il est très utile d'ajouter un axe « service technique ou BTO (Basic Technical Objects) » qui permet de disposer d'une vision nécessaire aux opérationnels pour pouvoir se comparer, réaliser des choix technologiques, mais aussi isoler certains coûts pour les relier directement aux services qui les utilisent. Le cas étudié démontre aussi qu'il est nécessaire d'ajouter un niveau d'analyse des données comptables (les modules de coûts). Ces modules de coûts permettent d'enrichir la comptabilité d'une analyse par fournisseur, par équipe, par lieu géographique ou technique (un datacenter par exemple) afin d'améliorer la lisibilité du modèle et de relier ces modules à l'inducteur adapté pour les déverser sur les activités ou sur les services techniques.

Enfin, en termes de gouvernance, la littérature et le cas étudié démontrent le besoin de transparence accrue dans le secteur bancaire dans lequel la piste d'audit entre les ressources et les services fournis est nécessaire. Ce besoin est encore plus crucial pour une DSI, surtout dans un contexte de mutualisation de moyens, car elle se doit de démontrer la bonne utilisation des ressources et de justifier la facturation de ses coûts. Le catalogue de services permet une contractualisation entre les acteurs, réduit les coûts de transaction et facilite la transformation. Le secteur bancaire est enclin à opérer des transformations majeures de ses activités informatiques car elles constituent un processus-clé, donc un avantage concurrentiel. Les moyens mis en œuvre permettent de tester la validité de nouvelles modélisations qui se sont avérées, dans le cas étudié, être un succès car elles ont permis de répondre aux différentes questions posées sur l'évaluation des coûts, la tarification et la refacturation des services. La mise en place d'un outil adapté de pilotage des coûts de la DSI renforce cet avantage concurrentiel car il éclaire les choix de technologie et d'organisation, et il permet une maîtrise de l'évolution des coûts afin de dégager une meilleure rentabilité de la banque, améliorant ainsi ses marges de manœuvre.

\section{Bibliographie}

AYADI N. \& BOUJELBENE Y. (2013), "The Influence of the Board of Directors on the Executive Compensation in the Banking Industry", Global Business \& Management Research, Vol. 5 (Issue 2/3), p. 83-90.

BESSON P. \& ROWE F. (2011), «Perspectives sur le phénomène de la transformation organisationnelle », Systèmes d'Information et Management, 16(1).

BOUAYNAYA W. \& BIDAN M. (2017, mai), « Une exploration qualitative du rôle des opérateurs du Cloud Computing dans l'acheminement des données des PME », Management \& Avenir (93), pp. 65-83.

CAM-I (1990), "The CAM-I Glossary of Activity-Based Management". 
CASEAU, Y. (2011), Processus et entreprise 2.0 innover par la collaboration et le lean management, Vol. 1, Paris, Dunod.

CICHOWLAS A. (2015), «Technologie et transformation des services bancaires : l'exemple de technovision de Capgemini ", Revue d'économie financière, 4 ( $\left.\mathrm{n}^{\circ} 120\right)$, pp. 35-54.

CIGREF (2014), "Modèle d'analyse et de benchmarking des coûts informatiques ", version 2014.

CIGREF (octobre 2009), "Modèle d'analyse et de benchmarking des coûts informatiques. Quels leviers pour piloter vos coûts? »

Commissariat général du Plan (Groupe présidé par Jacques Barraux) (1997), Entreprise et performance globale - Outils, évaluation, pilotage, Economica.

DANNON H., DUMOULIN R. \& VERNIER E. (2011, mars/avril), « Innovation et développement durable dans la banque : enjeux et perspectives », Gestion 2000, Vol. 28, Issue 2, p. 91-105.

DE LIMA P. (2005), «L'intégration du système financier en Europe », Revue de I'OFCE, 1(92), pp. 145-192.

ELIDRISSI D. \& A. (2010), "Contribution des systèmes d'information à la performance des organisations : le cas des banques ", La Revue des sciences de gestion, 1, pp. 55-61.

EZZI F. \& JARBOUI A. (2016, Jun), « Does innovation strategy affect financial, social and environmental performance? ", Journal of Economics, Finance and Administrative Science, Vol. 21 (Issue 40), pp. 14-24.

FORRESTER. (2012), « Montant des dépenses IT par secteur en 2012 », Récupéré sur www.forrester.com

HASNAOUI A. \& BIOT-PAQUEROT G. (2010, avril), « Systèmes d'information financiers et intégration des parties prenantes : proposition d'une grille de lecture ", Management \& Avenir (Issue 34), pp. 216-232.

JOHNSON H.T. \& KAPLAN R.S. (1987), Relevance Lost - The Rise and Fall of Management Accounting, Boston, Harvard Business Review Press.

LACOMBE I. (1997, décembre), thèse de doctorat en sciences de gestion, "L'application de la méthode ABC (Activity-Based Costing) au domaine des services ", Convention CIFRE avec Hewlett-Packard et l'IRG (Institut de Recherche en Gestion) de Paris XII.

LAVAYSSIÈRE B. (2015), "Les modèles économiques bouleversés par le digital », Revue d'économie financière, 4 ( $\left.n^{\circ} 120\right)$, p. 57-66. 
MEVELLEC P. (2017, juin), «La Design Science Research Methodology au service de la recherche en contrôle de gestion : application aux recherches sur les systèmes de coûts », Gérer \& Comprendre, pp. 62-78.

MORLEY C. \& BIA FIGUEIREDO M. (2016), Impact des processus de recherche sur les organisations. Le cas du domaine " systèmes d'information ", Revue française de gestion, 8 ( $\left.\mathrm{n}^{\circ} 261\right)$, pp. 21-38.

SAHUT J.-M. \& LANTZ J.-S. (2011), « Quel business model et performance pour les banques par Internet ? », Management \& Avenir (42), pp. 232-246.

SAIDANE D. \& MECHRI S. (2011, march/april), "Bank Transparency: A Microeconomic And Macroeconomic Assessment", Bankers Markets \& Investors, $\mathrm{n}^{\circ} 111$, pp. 54-62.

TREYER J. \& BROGNIART O. (2010), Modèle économique de la DSI - les clés de la performance, Hermès.

WEGMANN G. (2011), « Le déploiement d'un pilotage stratégique des coûts dans les services informatiques de deux groupes internationaux : perspective instrumentale et analyse comparative », Management \& Avenir, 4(44), pp. 78-96.

WILLIAMSON, O. E. (2002), "The Theory of the Firm as Governance Structure: From Choice to Contract", Journal of Economic Perspectives, vol. 16(n $n^{\circ}$ ), pp. 171-195. 